\title{
A Rare Case of Pseudomembranous Tracheitis Presenting as Acute Stridor in a Patient after Extubation
}

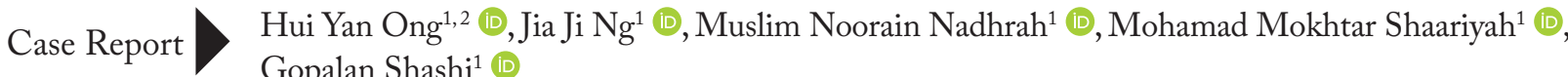 \\ ${ }^{1}$ Department of Otorhinolaryngology, Tengku Ampuan Rahimah Hospital, Ministry of Health, Klang, Selangor, Malaysia \\ ${ }^{2}$ Department of Otorhinolaryngology, University Malaya Medical Centre, Kuala Lumpur, Malaysia
}

Abstract

ORCID iDs of the authors: H.Y.0. 0000-0002-5610-1500; J.J.N. 0000-0001-7209-7196; M.N.N. 0000-0002-3157-6331; M.M.S. 0000-0001-7087-5812 G.S. 0000-0001-5536-6331.

Cite this article as: Ong HY, Ng J, Nadhrah MN, Shaariyah MM, Shashi G. A Rare Case of Pseudomembranous Tracheitis Presenting as Acute Stridor in a Patient after Extubation. Turk Arch Otorhinolaryngol 2020; 58(2): 130-2.

\section{Corresponding Author:}

Hui Yan 0ng, huiyan155@gmail.com

Received Date: 08.01 .2020 Accepted Date: 23.02.2020

Content of this journal is licensed under a Creative Commons Attribution 4.0 International License. Available online at www.turkarchotolaryngol.net
Pseudomembranous tracheitis is a rare life-threatening complication of endotracheal intubation. The exact mechanism of its formation is not well known, and it could mimic crusting or retained secretions in the trachea. We encountered a patient with history of recent intubation, presenting with acute stridor requiring emergency airway stabilization, and was eventually found to have pseudomembranous tracheitis.

Keywords: Stridor, intubation, tracheitis, airway

\section{Introduction}

Pseudomembranous tracheitis is a rare life-threatening complication of endotracheal intubation. The exact mechanism of its formation is not well known, and it could mimic crusting or retained secretions in the trachea (1). Patients may present with acute respiratory failure, and timely management including rigid bronchoscopy and removal of the membrane would ensure favorable outcome. We encountered a patient with a history of recent intubation, presenting with acute stridor requiring emergency airway stabilization, and was eventually found to have pseudomembranous tracheitis.

\section{Case Presentation}

A 68-year-old man, with underlying comorbidities, including diabetes mellitus and hypertension, presented to a district hospital with noisy breathing for two days and worsening shortness of breath for five days. He revealed a recent admission to the same hospital earlier, during which he was intubated for less than 24 hours for fluid overload symptoms. He was extubated well and discharged. Five days later, he started developing tightening of chest and shortness of breath, which were relieved slightly by resting and being propped up. He was brought to the emergency room a few times and was discharged after being nebulized. His symp- toms worsened and finally he was admitted to the same hospital when he started developing noisy breathing. He was given steroid therapy and nebulized in the ward, but there was no improvement. He was eventually referred to our center by the district hospital for ENT assessment.

Examination by the ENT team showed that he was in distraught with loud biphasic stridor. Oxygen saturation was maintained despite the labored breathing. He was immediately taken to the operating room for further assessment in a controlled environment.

Flexible nasoendoscopy was performed promptly. Thick crusting and secretions were seen to have occupied almost the entire subglottic region. The bilateral vocal cords were equal and mobile, with no mass seen. Rigid bronchoscopy was performed via a bronchoscope after the patient was sedated and ventilated. The pseudomembrane-like structure with crusting and secretions was removed (Figure 1). Inflammation of the surrounding tracheal wall was also noted post removal of the crusting (Figure 2). He achieved complete resolution of symptoms after the procedure and a course of intravenous antibiotics. Flexible nasoendoscopy performed one month post procedure revealed normal and healthy tracheal wall with no tracheal stenosis. 
The patient recovered well and was discharged by our clinic. He has given us verbal consent for the publication of his case history; his identity is preserved and remains confidential.

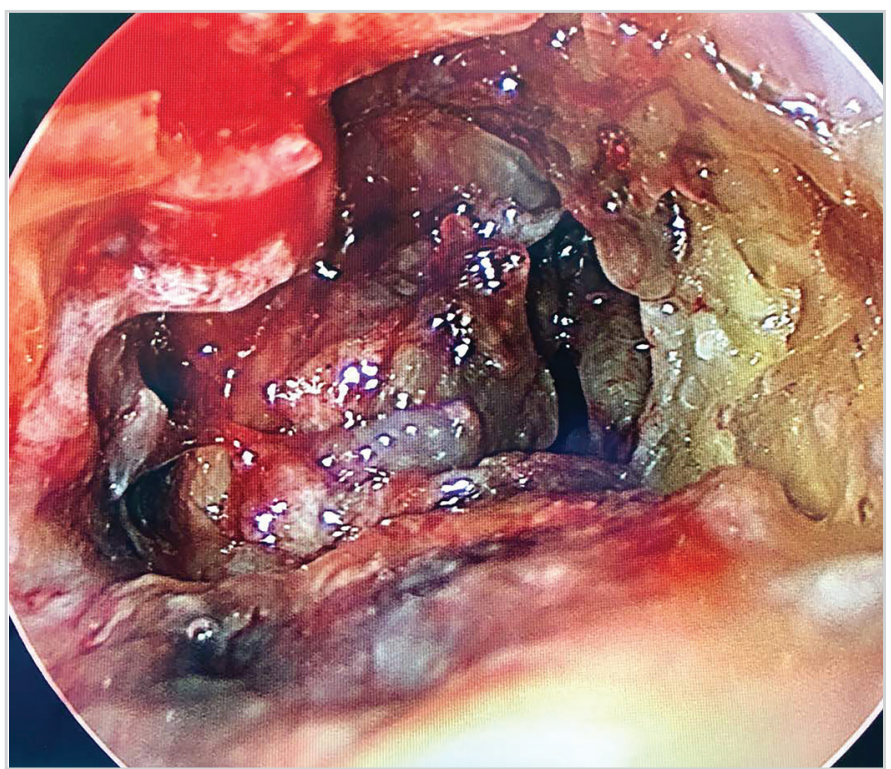

Figure 1. Rigid bronchoscopy revealed that almost the entire subglottic region was covered by thick crusting and pseudomembrane-like structure

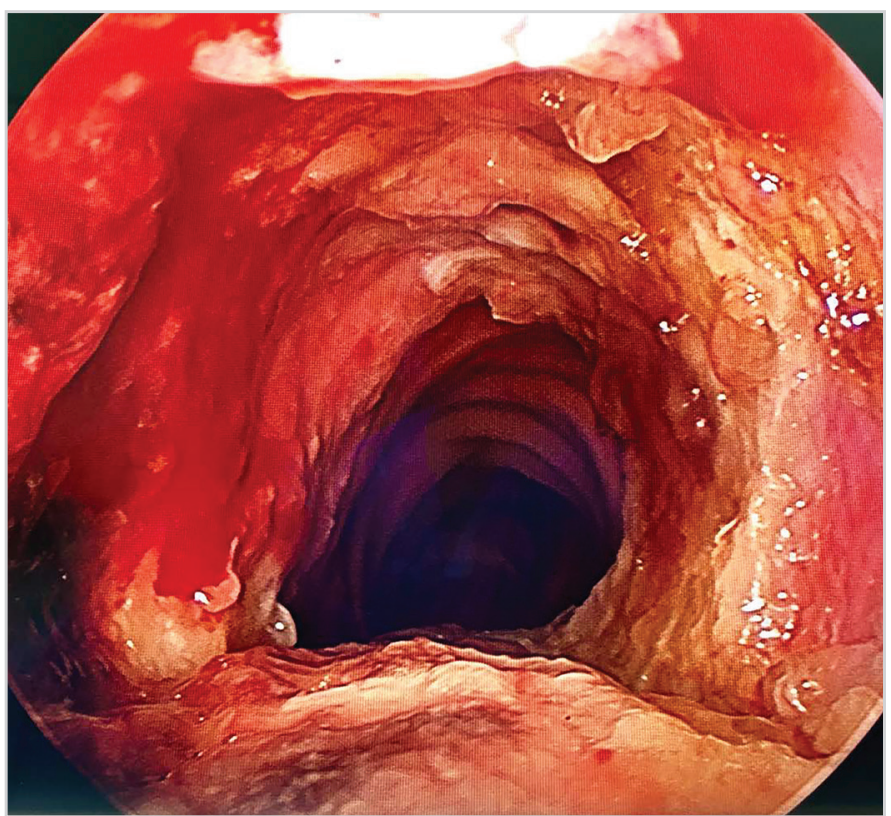

Figure 2. Post removal of the crusting and the pseudomembranelike structure, the surrounding tracheal wall appeared inflamed and sloughy

\section{Main Points}

- Pseudomembranous tracheitis is a rare life-threatening complication of endotracheal intubation.

- Patients with post extubation stridor not responding to standard steroids therapy requires further evaluation and other uncommon complications should be considered.

- Clinical suspicion and prompt rigid bronchoscopy will ensure favourable outcomes.

\section{Discussion}

Endotracheal intubation may occasionally lead to tracheal complications such as tracheal stenosis, tracheomalacia, or granuloma (2). Pseudomembranous tracheitis following endotracheal intubation is rare and often underdiagnosed (1). It was reported that one out of ten patients who suffered from this life-threatening complication succumbed due to acute asphyxiation (2). The exact mechanism of its formation is not well studied, and it could mimic crusting or retained secretions in the trachea (1).

There are many postulations regarding the formation of pseudomembrane (3). The subglottic region is the narrowest part of the adult larynx and is vulnerable to injury during intubation (3). Following an injury, desquamated epithelial cells may accumulate to form a membranous-like structure (3). It has also been postulated to be the initial phase of the development of tracheal stenosis, whereby the inflated endotracheal cuff causes tracheal mucosa and submucosa necrosis (3).

Patients may present with non-specific symptoms such as dyspnea or stridor, few hours to even several days post extubation (4). It is usually misdiagnosed as laryngeal or subglottic oedema. Subglottic oedema usually manifests within one hour post extubation and may progress in the next four hours, and then eventually resolve after 24 hours (5).It is usually responsive to steroid therapy (5). In our case, the patient was only intubated for a brief period of less than 24 hours, and there was no sign of infection. His symptoms developed five days post extubation and worsened over the next few days, with no improvement after standard steroid therapy.

Rigid bronchoscopy and removal of the pseudomembrane are the gold standard of treatment for this condition, though spontaneous expectoration of the pseudomembrane has also been reported (5). Imaging such as computed tomography of the neck and the thorax may be useful when the cause of the respiratory symptom is not identifiable (3), provided that the patient is stable with no impending airway compromise.

In our case, timely flexible nasoendoscopy provided the clue and prompt rigid bronchoscopy confirmed the diagnosis. The patient recovered well post removal of the pseudomembrane and completed a course of intravenous antibiotics.

\section{Conclusion}

Pseudomembranous tracheitis should be considered in patients who present with post extubation stridor and do not respond to standard steroid therapy. Clinical suspicion and prompt rigid bronchoscopy with removal of the pseudomembrane will result in favorable outcome.

Informed Consent: Informed consent was obtained from the patient.

Peer-review: Externally peer-reviewed.

Author Contributions: Literature Search - H.Y.O., J.J.N., M.M.N.; Writing - H.Y.O.; Critical Reviews - M.M.S., G.S. 
Acknowledgement: The authors would like to thank the Director General of Health Malaysia for the permission to publish this paper.

Conflict of Interest: The authors have no conflicts of interest to declare.

Financial Disclosure: The authors declared that this study has received no financial support.

\section{References}

1. Manassero A, Ugues S, Bertolaccini L, Bossolasco M, Terzi A, Coletta A. A very early stage of obstructive fibrinous tracheal pseudo-membrane formation. J Thorac Dis 2012; 4: 320-2.
2. Deslée G, Brichet A, Lebuffe G, Copin MC, Ramon P, Marquette $\mathrm{CH}$. Obstructive fibrinous tracheal pseudomembrane. A potentially fatal complication of tracheal intubation. Am J Respir Crit Care Med 2000; 162: 1169-71. [Crossref]

3. Sehgal IS, Dhooria S, Bal A, Aggarwal AN, Behera D, Agarwal R. Obstructive fibrinous tracheal pseudomembrane after endotracheal intubation. Respir Care 2016; 61: 1260-6. [Crossref]

4. Niusha S, Salehi F, Behgam Shadmehr M. Obstructive tracheal pseudomembrane. Arch Iran Med 2017; 20: 714-5.

5. Fiorelli A, Vicidomini G, Messina G, Santini M. Spontaneous expectoration of an obstructive fibrinous tracheal pseudomembrane after tracheal intubation. Eur J Cardiothorac Surg 2011; 40: 261-3. [Crossref] 